\title{
L'APPEL DU NÉANT SUBSTANTIEL : CLAUDE SIMON ET LA MORT TELLURIQUE
}

\section{[...] car tu es poussière, et tu retourneras dans la poussière.}

La Genèse, 3 17-19

La présence - ou plutôt l'omniprésence - des quatre éléments de la nature marque profondément les romans de Claude Simon, et surtout ceux publiés à partir de 1957. Cette date indique un tournant dans son écriture, exprimé par le rejet de la structure traditionnelle du genre romanesque en faveur des expérimentations formelles caractéristiques du nouveau roman. Avec Le Vent (1957), Simon se lance dans une composition brouillée et fragmentaire, transgressant les règles de linéarité, de causalité et de cohérence de l'intrigue. Par de longues phrases sinueuses à la ponctuation lacunaire, l'écrivain semble vouloir saisir et transcrire la mémoire, et surtout ses souvenirs relatifs à la Deuxième Guerre mondiale à laquelle il a participé en tant que brigadier de dragons ${ }^{1}$. La technique du flux de la conscience lui permet de revivre et de retravailler certaines images traumatisantes, comme celle de la mort de son colonel lors de la déroute de l'armée française en Flandres, qui hanteront, en motifs récurrents, ses futurs romans. C'est aussi à l'expérience néfaste de la guerre que l'on doit sa conception pessimiste du monde hostile et de l'existence humaine, inaccomplie et absurde. Dans un tel décor lugubre, les

${ }^{1}$ Toutes les informations biographiques fournies dans l'article proviennent de la biographie de l'écrivain par Mireille Calle-Gruber, Claude Simon. Une vie à écrire, Seuil, Paris 2011. 
romans simoniens portent témoignage d'une tension permanente entre la vie et la mort, avec une prédilection pour cette dernière. Qui plus est, les quatre puissances de la nature contribuent, chacune à sa façon, à l'anéantissement de l'univers et des êtres vivants. En bref, chez Simon, la dégradation globale se produit et se propage au moyen des éléments.

Dans le présent article, nous nous bornerons à étudier seulement l'élément tellurique, afin de montrer son implication, en tant que conditio sine qua non, dans le processus d'agonie des organismes vivants.

Claude Simon est l'auteur d'une trentaine d'œuvres littéraires et photographiques; nous avons choisi de nous concentrer sur ses sept premiers récits parus aux Éditions de Minuit et appartenant à sa période néo-romanesque (les années 1957-1971) ${ }^{2}$. Soit, dans l'ordre de publication : Le Vent (1957), suivi de L'Herbe (1958), de La Route des Flandres (1960), du Palace (1962), de Histoire (1967), de La Bataille de Pharsale (1969) et des Corps conducteurs (1971) ${ }^{3}$. Pour notre analyse, nous proposons une approche pluridisciplinaire, puisant non seulement dans la critique littéraire, mais aussi dans la philosophie, la poésie et même dans la photographie. Nous allons donc nous inspirer, entre autres, de la théorie des quatre éléments de Gaston Bachelard et plus particulièrement de ses deux ouvrages consacrés aux rêveries liées à la terre ${ }^{4}$, ainsi que des travaux de nombreux chercheurs, tels Michèle Raclot, Christine Genin, Catherine Haman-Dhersin, Alastair Duncan, Ghislain Bourque, Jean-Yves Laurichesse, Sjef Houppermans, Irène Albers, Jean-Claude Vareille et Philippe Crippa. Nous allons aussi faire référence à l'ouvrage théorique de Roland Barthes sur la photographie, au poème célèbre d'Arthur Rimbaud et à la philosophie du présocratique Zénon d’Élée.

${ }^{2}$ N.B. Nous ne parlerons que des œuvres romanesques de Simon et omettrons ses deux ouvrages qualifiés par Alain Cresciucci de «non romanesques », c'est-à-dire consacrés à la peinture. Il s'agit des Femmes. Sur vingt-trois peintures de Joan Miró (1966) et d'Orion aveugle (1970), ce dernier étant d'ailleurs repris en quasi totalité dans Les Corps conducteurs. Cf. A. Cresciucci, Claude Simon. La Route des Flandres, Klincksieck, Paris 1997, p. 26.

${ }^{3}$ Les références à ces œuvres sont fournies dans la suite de l'article sous la forme des abréviations suivantes :

VEN - Le Vent. Tentative de restitution d'un retable baroque, Minuit, Paris 1975 [1957]. HER - L'Herbe, Minuit, Paris 1958.

FLA - La Route des Flandres, Minuit, Paris 1960.

PAL-Le Palace, Minuit, Paris 1962.

HIS - Histoire, Minuit, Paris 1967.

PHA - La Bataille de Pharsale, Minuit, Paris 1969.

COR - Les Corps conducteurs, Minuit, Paris 1971.

${ }^{4} \mathrm{G}$. Bachelard, La Terre et les rêveries du repos : Essai sur les images de l'intimité, Corti, Paris 2004 [1946], et idem, La Terre et les rêveries de la volonté : Essai sur l'imagination de la matière, Corti, Paris 2007 [1948]. 


\section{LA MORT DANS LE CORPS, LA MORT DANS L’ÂME}

Lorsque Christine Genin évoque le pourrissement universel des êtres exposés dans les œuvres de Simon $^{5}$, elle se réfère à une atmosphère contaminée par la mort tellurique qui traverse l'univers romanesque de l'écrivain. L'« infection » chtonienne se manifeste par trois voies, en l'occurrence : indirecte, qui opère sur la métaphorique de la couleur ; directe, qui suppose un contact physique avec l'élément; et intermédiaire, qui unit la dimension métaphorique au contact réel et se résume dans la notion de l'immobilisme. Analysons dans l'ordre chacun de ces trois modes d'appel du néant chtonien.

Le processus d'assimilation des personnages simoniens à l'élément chtonien, dont parle l'aphorisme célèbre issu de la Genèse et repris en exergue du présent article, s'effectue, en apparence, sans même entrer en contact direct avec la terre. Ainsi, seule la couleur terreuse suffit à marquer l'appartenance d'un être vivant à l'élément. En guise d'exemple, songeons à une scène de La Bataille de Pharsale, dans laquelle les ouvriers agricoles attendent leur rémunération devant la porte du bureau d'O., l'oncle du narrateur :

[L]a mort dans l'âme la peine de mort. Je ne savais pas encore pour moi silhouettes grisâtres indistinctes attendant silencieusement dans la pénombre verte revêtues de choses pendantes informes terreuses molles exhalant cette acre odeur de fatigue de sueur morts de fatigue. $(P H A, 18)$

Les laboureurs sont présentés comme des figures grisâtres vêtues de loques terreuses. Leur peau, comme le précise Simon à la page précédente, est de couleur « gris verdâtre » $(P H A, 17)$, évidemment malsaine. La description extérieure des travailleurs n'est, au fond, qu'une illustration de leur état pitoyable et la prédiction de leur destin, celui-ci menant directement à la terre (ou, plus exactement, sous la terre). Il semble que les ouvriers n'aient qu'à attendre la réalisation de la métaphore contenue dans l'expression « mort de fatigue », qui mettra fin à cette vie misérable, imprimée dans leurs corps exténués.

Tout comme les travailleurs agricoles, les soldats simoniens portent une empreinte corporelle de leur condition pénible. La constante menace de mort au champ de bataille semble les réconcilier d'avance avec la terre. Il apparaît que le substantif même " soldat » témoigne d'un rapport étroit que le guerrier entretient avec l'élément tellurique. Vu que la guerre constitue, selon Michèle Raclot, une " forme accélérée de la décomposition de l'être ${ }^{6}$, les guerriers, amaigris et épuisés, ressemblent déjà à des squelettes. Les haillons sur leurs corps découvrent

${ }^{5}$ C. Genin, Écheveau de la mémoire, mémoire : « La Route des Flandres » de Claude Simon, Champion, Paris 1997, p. 95.

${ }^{6} \mathrm{M}$. Raclot, «Une constante de l'imaginaire simonien : l'obsession de la décomposition et du pourrissement dans La Route des Flandres », [dans :] R. Ventresque (dir.), "La Route des Flandres » : Claude Simon, Ellipses, Paris 1997, pp. 74-87; p. 74. 
« les peaux brûlées, d'une couleur terreuse, sous lesquelles saille chaque tendon, chaque muscle et chacun des os [...] comme des écorchés des planches d'anatomie » $(C O R, 183)$. L'adjectif " terreux » véhicule une vision déplorable de la profession militaire. Songeons encore au camp où stationnent trois protagonistes de La Route des Flandres, les compagnons-soldats Georges, Blum et Iglésia. Penchés au-dessus d'un petit feu allumé clandestinement dans le but de se préparer quelque pâte farineuse à manger, les hommes observent les autres guerriers faméliques errant sur le terrain militaire,

[u]niformément revêtus de ces défroques, couleur de bile, de boue, comme une sorte de moisissure, comme si une espèce de pourriture les recouvrait, les rongeait, les attaquait encore debout, d'abord par leurs vêtements, gagnant insidieusement : comme la couleur même de la guerre, de la terre, s'emparant d'eux peu à peu, eux, leurs visages terreux, leurs loques terreuses, leurs yeux terreux aussi, de cette teinte sale, indistincte qui semblait les assimiler déjà à cette argile, cette boue, cette poussière dont ils étaient sortis et à laquelle, errants, honteux, hébétés et tristes, ils retournaient chaque jour un peu plus. (FLA, 172)

Dans cet univers monochromatique assimilant la guerre à la terre, tout tend à s'unifier dans la forme et la couleur. Citons à cette occasion Roland Barthes qui constate, dans sa Chambre claire, que dans le cas de la photographie, « la couleur est un enduit apposé ultérieurement sur la vérité originelle du noir et blanc. La couleur est $[\ldots]$ un postiche, un fard (tel celui dont on peint les cadavres) $»^{7}$. Chez Simon, c'est l'inverse, car le monde extérieur — primairement en couleur - tend à la monochromie de la mort, s'approprie déjà symboliquement ce fard cadavérique qu'est la couleur brunâtre de la terre.

Le même passage de La Route des Flandres montre également comment se propage la métaphorique « infection » terrestre qui finit par envahir le corps entier des soldats. L'environnement boueux du camp militaire communique aux haillons que sont devenus, à force d'usure, les uniformes militaires, la saleté et la moisissure générale. Le contact avec de tels vêtements ne peut qu'altérer, par la suite, la couleur naturelle de la chair, voire des yeux des personnes qui les portent. Du niveau extérieur, on passe donc à l'intérieur de l'organisme, pour observer l'influence néfaste de l'élément. La peau teintée de brun devient ce que l'écrivain Pierre Loti qualifie de " défroque de chair et d'os, destinée à faire de l'humus pour les générations futures $»^{8}$. Le corps sous l'emprise du chtonien n'est plus considéré que dans son proche devenir : l'humus, l'argile, la poussière. La couleur l'emporte donc sur la forme ou plutôt, elle décide de la forme : l'homme cesse d'être ce qu'il est de par sa nature physique et s'assimile à la matière terrestre ; ses contours se confondent progressivement avec l'environnement, comme si le passage de l'organique à l'inorganique s'effectuait in vivo, sans passer par l'état intermédiaire de la mort.

${ }^{7}$ R. Barthes, La Chambre claire. Note sur la photographie, Gallimard, Paris 1980, pp. 127-128.

${ }^{8}$ P. Loti, Fleurs d'ennui : récits et nouvelles, Passage du Marais, Paris 2003, $1^{\text {re }}$ rééd. depuis 1882, p. 22. 


\section{VIERGE, VAMPIRIQUE ET VORACE}

Le contact direct avec le chtonien se voit, lui aussi, chargé de valorisations négatives. La scène tirée de La Route des Flandres qui montre Georges cherchant de l'aide auprès de la Terre-Mère, est particulièrement émouvante. Le protagoniste du roman se rappelle le moment où il arrive avec d'autres soldats sur un lieu de campement provisoire au milieu d'un pré et, souffrant terriblement de la faim, se jette au sol afin de manger de l'herbe fraîche. Celle-ci est naturellement incomestible : son " jus vert et âpre " $(F L A, 259)$ laisse une impression désagréable dans la bouche et, comme pour combler le malheur de Georges, « un brin effilé [lui] coup[e] la langue comme un rasoir [le] brûlant» $(F L A, 259)$. Quoiqu'il s'avère impossible d'apaiser sa faim grâce à l'abondante végétation, le désespoir pousse le protagoniste à feindre le contraire : "Les chevaux en mangent bien pourquoi pas moi j'essayais de m'imaginer me persuader que j'étais un cheval » $(F L A, 258)$. Face à une réalité brutale, les belles métaphores de la terre nourricière échouent ; censée subvenir aux besoins de sa création, cette alma mater sélective ne favorise que les animaux herbivores. Pour les hommes, elle reste indifférente sinon hostile ${ }^{9}$. Catherine Haman-Dhersin compare la terre telle qu'elle est représentée chez Simon plutôt à un « vampire assoiffé qu['à] un objet nourricier » ${ }^{10}$. Par ailleurs, les adjectifs choisis par l'auteur de La Route des Flandres pour décrire la prairie sur laquelle se jette Georges ne sont pas non plus sans importance : lorsque le protagoniste la qualifie de " vierge et impolluée » (FLA, 258), cette glorification au niveau esthétique laisse toutefois sous-entendre une ambivalence. Anthropomorphisée, la terre fait penser à une femme intouchée, alors en quelque sorte stérile. En poursuivant cette direction associative, si la femme n'a jamais enfanté, elle ne s'inscrit pas dans le contexte réellement nutritif, mais demeure une promesse de boisson revigorante. Dans cette lumière, la virginité de la prairie devient l'antithèse de la maternité véritable ${ }^{11}$, ce qui sonne amèrement dans la tête du soldat affamé qui y cherche instinctivement de la nourriture.

Ayant compris la futilité de son action, Georges s'immobilise sur l'herbe, épuisé par cette ultime tentative de secours. Mais seront déçus ceux à qui leur imagination suggère ici un tableau paisible tout juste sorti du célèbre poème d'Arthur Rimbaud, Le Dormeur du val. Le sonnet dépeint le décor idyllique d'une vallée

${ }^{9}$ C'est d'ailleurs Simon lui-même qui confirme, dans un entretien avec Mireille Calle, sa vision de l'élément : « si la terre garde une empreinte des hommes (cultures, carrières, grands travaux de terrassement), elle est parfaitement indifférente à leur souffrances, que ce soit celles de champs de bataille ou les autres » (C. Simon dans un entretien avec Mireille Calle, [dans :] idem, L'Inlassable $r^{\prime a}{ }_{e}$ encrage du vécu, La Différence, Paris 2011, p. 133).

${ }^{10}$ C. Haman-Dhersin, Les Paysages de Claude Simon, Septentrion, Villeneuve d'Ascq 2012, p. 54.

${ }^{11}$ Laissons à part le discours de la Bible selon lequel la Vierge Marie réunit deux aspects de la féminité à la fois : la maternité et la virginité. 
ensoleillée et parcourue par un ruisseau argentin, au bord duquel est étendu un soldat mort, entouré d'une végétation fleurissante. En fait, rien de plus éloigné $\mathrm{du}$ « dormeur » rimbaldien que ce misérable personnage de Georges. Chez Simon, la terre ne laissera pas le pitoyable cavalier se reposer tranquillement afin de regagner des forces. À l'instant même où celui-ci se couche sur l'herbe, les insectes voraces apparaissent, pareils à d'innombrables agents telluriques dont le rôle est de décomposer le corps inerte pour le transformer en une masse inorganique qui servira ensuite de pâture à la terre anthropophage.

[J]e gisais mort au fond du fossé dévoré par les fourmis mon corps tout entier se changeant lentement par l'effet d'une myriade de minuscules mutations en une matière insensible et alors se serait l'herbe qui se nourrirait de moi ma chair engraissant la terre. (FLA, 259)

La glèbe carnivore, quel concept paradoxal ! Pourtant, la même image ressort des Corps conducteurs où la végétation et les fourmis, animaux terrestres par excellence, consument les cadavres laissés par la guerre :

Le chemin suivi est jalonné par les carcasses des moules déplacées et abandonnées dont les restes sont aussitôt dévorés par les fourmis. Sous leur grouillement rougeâtre apparaissent très vite les os couleur d'ivoire, le berceau des côtes, les crânes aux longues dents jaunes. En peu de temps, les herbes, les lianes, la végétation exubérante en prennent possession, s'insinuent dans les interstices, les cavités, les orbites vides, s'enroulent autour des os encore puants. $(C O R, 184)$

La cruelle vision simonienne - ou peut-être simplement naturaliste -, n'a donc rien à voir avec le romantisme du poète maudit. Le soldat Georges est traité simplement de dépouille encore vivante, prête à être déchiquetée et retournée à la terre au plus vite.

Ce n'est pas un hasard si l'herbe est choisie par Simon à la fois pour constituer le fond sur lequel se déroule un drame funeste, et pour jouer le rôle d'agent principal de ce drame même. Le roman intitulé L'Herbe est avant tout une œuvre portant sur l'agonie tellurique sous ses formes multiples ${ }^{12}$. L'action du récit se passe dans une maison à la campagne, où habitent Pierre, homme opulent écrasé par son propre poids, Marie, une vieille tante agonisante, Sabine cachant sa vieillesse sous un visage grotesquement fardé, et la jeune Louise, cherchant ce que Georges Bataille nomme « la petite mort» dans un acte sexuel. Et tout cela dans une atmosphère imprégnée de l'âcre odeur des fruits en putréfaction. Si chacun de ces personnages affronte plus ou moins directement la mort, la figure de Marie représente cette dernière de la façon la plus évidente. Son agonie interminable fusionne les impressions auditives et les relents de pourriture : «l'écho du râle arrivant maintenant par l'extérieur [de la fenêtre] avec l'odeur cadavérique et fermentée des milliers de poires en train de pourrir sur la terre noire » $(H E R, 114)$. Mais elle n'est pas la seule à entendre l'appel mortifère du tellurique. Même un corps

12 Dans la postface du livre en question, Alastair Duncan avoue que c'est une œuvre écrite « sous le signe de la mort » (A. Duncan, « Lire L'Herbe », [dans :] C. Simon, L'Herbe, Minuit, Paris 1986). 
jeune et plein de vie comme celui de Louise, une fois exposé au contact avec le sol, sympathise avec sa dimension inorganique. Dans le passage de L'Herbe cité ci-dessous, la femme succombe à la fraîcheur ensorcelante du pré et s'immerge complètement dans l'élément. Après la rencontre avec son amant, Louise reste allongée sur le sol, " sentant l'odeur, la senteur végétale, humide, la pénétrant, comme si ce n'était pas de l'herbe foulée qu'elle s'exhalait mais des profondeurs, du sein même de la terre, pensant : 'Voilà. Je suis morte' ( $H E R, 174)$. Une centaine de pages plus loin, la même scène revient, cette fois-ci avec davantage de détails :

[Louise] couchée maintenant de tout son long au sol, adhérant au sol, enfonçant, enfouissant son visage dans l'herbe fraîche, comme pour l'y imprimer, respirant longuement l'odeur puissante et âcre d'herbe et de terre mêlées [...] s'emplissant toute entière de l'odeur végétale et pure, puis se relâchant, s'abandonnant, toujours allongée sur le ventre, mais la tête tournée sur le côté, pouvant maintenant sentir s'imprimer dans sa joue les croisillons d'herbe écrasée. (HER, 254)

Comme le commente Ghislain Bourque, le « corps tout entier [de Louise], non seulement respire l'herbe, mais l'habite. Fondue à la terre, elle se laisse envahir par elle, l'herbe s'imprimant $[\ldots]$ sur son corps $»^{13}$. C'est par l'intermédiaire de l'odeur que l'élément s'insinue à l'intérieur de sa chair. À Jean-Yves Laurichesse de le confirmer : "Par l'olfaction s'accomplit un échange parfait entre le corps et la terre, une osmose totale, qui conduit à un dépassement quasi panthéiste de l'opposition entre la vie et la mort ${ }^{14}$. Ce dépassement de l'opposition binaire animé/inanimé dans un organisme vivant - pas forcément celui de l'homme - évoque la rêverie bachelardienne de la racine, exemple végétal « de la synthèse active de la vie et de la mort ». Bachelard explique que « [1]a racine n'est pas enterrée passivement, elle est son propre fossoyeur, elle s'enterre, elle continue sans fin à s'enterrer $»^{15}$. Simon reprend ce motif végétalien pour parler de Georges à demi-mort, mangeant ou plutôt intériorisant la terre, son corps exhalant déjà, en signe de son destin, " cette âcre et forte odeur de racine, de mandragore » (FLA, 259).

Remarquons que jusque-là, le « processus d'uniformisation de l'homme et de la terre $»^{16}$, pour emprunter l'expression à Sjef Houppermans, s'accomplit doucement, sans aucune violence de déchirement ou de morcellement infligée au corps. L'assimilation en entier à l'élément chtonien, à l'instar de Louise qui se fond doucement dans le sol herbu, s'accorde avec la vision poétique de Rimbaud. On est à

${ }^{13}$ G. Bourque, «La parabole : analyse végétale de L'Herbe », Études Littéraires 9 (1), 1976, pp. $161-187$; p. 187.

14 J.-Y. Laurichesse, La Bataille des odeurs. L'Espace olfactif des romans de Claude Simon, L'Harmattan, Paris 1998, p. 41.

${ }^{15}$ G. Bachelard, La Terre et les rêveries du repos..., pp. 311-312.

${ }^{16}$ S. Houppermans, "La Rose des vents simonienne », [dans :] Claude Simon géographe, Actes du colloque à Toulouse (26-27/05/2011), sous la dir. de J.-Y. Laurichesse, Garnier, Paris 2013, pp. 241-253; p. 244. 
deux pas d'un enterrement. Et peu importe que celui-ci soit métaphorique, comme dans le cas de Louise qui se repose après un acte amoureux, ou réel, dans le cas de Marie, pour qui l'ensevelissement serait un véritable coup de grâce qui mettrait fin à sa longue agonie. Nous n'allons toutefois pas développer ici la thématique de l'enterrement chez Simon ; contenterons-nous de signaler que ce motif méritera une étude à part.

\section{ACHILLE IMMOBILE À GRANDS PAS ${ }^{17}$}

Et si l'immobilité d'un organisme préfigurait son dernier repos ? Là où le contact métaphorique avec l'élément terrestre rencontre le contact réel, la mort semble être le résultat d'une inertie prolongée. Le protagoniste de La Route des Flandres partage ses observations de cadavre non-enterré : " peut-être était-ce une pure question d'immobilité et alors on redevenait simplement un peu de craie, de sable et de boue » $(F L A, 229)$. Du point de vue physique, la matière en état de repos se caractérise par une absence de mouvement, mais signifie-t-elle aussi l'absence de vie ? Simon situe-t-il l'immobilité dans le contexte du repos temporel davantage que dans celui du repos éternel ?

Irène Albers rappelle ici la figure du philosophe grec présocratique, Zénon d'Élée, dont la philosophie visait à prouver qu' « il n'y avait pas de véritable mouvement, $[\ldots]$ toute forme de mouvement et de passage se décomposait en une série d'états et de moments immobiles ». Chez Simon, continue Albers, " mouvement et immobilité s'unissent dans la synthèse paradoxale d'une 'immobilité à très grande vitesse' " ${ }^{18}$ qui apparaît, paradoxalement, comme le superlatif du mouvement. Ce n'est pas par hasard que Jean-Claude Vareille avance le concept de l' « énergétique de l'immobile » dans les œuvres simoniennes : «L'immobilité, écrit-il, est l'énergie condensée, densifiée, hypertrophiée, amenée à l'état explosif. D'où l'obsession du fantasme éléatique : l'immobilité n'est que la vérité superlative de la vitesse ${ }^{19}$. La meilleure illustration de ces propos se trouve dans le fragment suivant de L'Herbe : « le temps [...] solidifié, semblable à une foudroyante condensation de la vitesse » se voit capable de «transformer la vitesse même en sa représentation immobile» $(H E R, 18)$.

La simonienne « inclination à l'immobilisme $»^{20}$, pour reprendre l'expression de Philippe Crippa, est due, au niveau formel, au primat de l'instant dans

${ }^{17}$ Ce vers emprunté au poème Le Cimetière marin de Paul Valéry se voit repris par Simon en épigraphe pour la première partie de La Bataille de Pharsale.

${ }^{18}$ I. Albers, Claude Simon, moments photographiques, Septentrion, Villeneuve d'Ascq 2007, p. 112.

19 J.-C. Vareille, « À propos de Claude Simon : langage du cosmos, cosmos du langage », Études littéraires 1, 1984, pp. 13-44; p. 123.

${ }^{20}$ P. Crippa, «Sensations et perceptions dans La Route des Flandres », [dans :] B. Bonhomme et al., Claude Simon, Actes n 3, Le Texte et l’Édition, Dijon 1998, pp. 19-53 ; p. 46. 
la narration de l'auteur de La Route des Flandres. Si le concept de la temporalité figée, manifesté dans l'écriture simonienne par le suremploi des participes présents, est abordé dans plusieurs études ${ }^{21}$, il n'en va pas de même avec le niveau sémantique où règnent les images métaphoriques de l'immobilité. L'imagerie symbolique dominante, chez Simon, s'associe essentiellement aux idées de froid glaçant. Ainsi, pour reprendre les paroles de Crippa, un "phénomène de solidification traverse l'œuvre [simonienne] : certains épisodes semblent se dérouler 'comme au ralenti', le temps se substantifie en un 'lent glacier' $\gg 2$. L'exemple du glacier dont se sert le chercheur est d'ailleurs beaucoup plus significatif si on le replace dans son contexte. Examinons donc la scène entière de La Route des Flandres où deux prisonniers de guerre, Georges et Blum, échangent quelques paroles, ou plutôt quelques sons qui passent difficilement pour du langage :

Les deux voix sans visage alternant se répondant dans le noir sans plus de réalité que leur propre son, disant les choses sans plus de réalité qu'une suite de sons, continuant pourtant à dialoguer : au commencement seulement deux morts en puissance, puis quelque chose comme deux morts vivants, puis l'un d'eux véritablement mort, et l'autre toujours vivant $[\ldots]$, et tous deux [...] pris, enserrés par cette chose à la fois immobile et mouvante qui rabotait lentement sous son poids la surface de la terre (et peut-être était-ce cela que Georges continuait toujours à percevoir, comme un glissement, un raclement imperceptible, monstrueux et continu derrière le menu et patient piétinement des sabots : cette olympienne et froide progression, ce lent glacier en marche depuis le commencement des temps, broyant, écrasant tout, et dans lequel lui semblait les voir, lui et Blum, raides et glacés, [...] intacts et morts parmi la foule des fantômes. (FLA, 278-279)

L'existence des deux personnages se voit remise en question, leurs voix irréelles ne produisant «qu'une suite de sons » non reconnaissable comme un langage. Les hommes oscillent entre le royaume des vivants et celui des morts, piégés par (dans ?) cette chose immense et innommée mais saisie par la métaphorique du glacier, qui engloutit et emporte le monde dans son mouvement paradoxal frôlant l'immobilité.

Crippa ajoute que la " répétition d'une même perception [...] emprisonne le sujet percevant dans une cyclicité qui le laisse perplexe $»^{23}$. En conséquence, l'écriture simonienne, qui reprend et retravaille inlassablement les mêmes motifs, piège ses personnages, à la manière d'une masse glaciale. C'est pire encore si l'homme, de nature soumis aux changements et au mouvement incessant, se veut observateur. Selon Crippa,

${ }^{21}$ Voir la notion de « l'éternel présent de l'écriture » de Philippe Richard, de « toujours-présent-de-l'écriture » de Mireille Calle-Gruber, ou encore de « présent mémoriel » de Vincent Gracy (P. Richard, «Claude Simon, de l'ekphrasis à l'icône » (Essais critiques), Acta fabula, vol. 16, $\mathrm{n}^{\circ} 4$, 2015, p. 13 ; M. Calle-Gruber, « Claude Simon : le temps, l'écriture », Littérature 11, 1991, pp. 31 42 (p. 33) ; V. Gracy, « Claude Simon — présent mémoriel, passé sur-vivant », Secousse 11, 2013).

${ }^{22}$ P. Crippa, op. cit., p. 46.

${ }^{23}$ Ibidem, pp. 46-47. 
pour que l'acte perceptif se pose en véritable révélateur du monde, il convient que l'observateur soit lui aussi immobile. Ce dernier se métamorphose alors en un pivot inflexible autour duquel graviteraient toutes ses perceptions. Plus il est immobile, plus il emmagasine le monde perçu dans une volonté de le concentrer, de le ramasser ${ }^{24}$.

L'observateur simonien s'approche de l'idéal lorsqu'il supprime le moindre mouvement corporel, soit-il contrôlable ou involontaire, en faveur des cinq sens.

Dans l'amour, autrement que dans l'observation, la perception sensorielle ainsi que le corps restent perplexes. Simon plonge les amants épuisés par l'acte sexuel dans une insensibilité et une inertie avoisinant la mort. Dans Histoire, l'auteur expose deux personnages allongés sur le lit et éclairés par la lumière bleuâtre de la lune, prêtant à leur peau un ton morbide :

lui et elle gisants étendus raides ou plutôt roides sur ce lit et sans doute la même lueur laiteuse répandue sur le drap les deux corps nus peut-être puisque c'était l'été laiteux de la même matière froide inanimée et polie que les plis du drap repoussé ou peut-être elle ayant ramené le drap par un réflexe non de pudeur mais de désespoir couvrant cachant ou plutôt ensevelissant déjà ce corps [...] dans cette vague lueur décolorée qui se répandait sur sa peau sous laquelle semblait circuler non pas du sang mais une pale sève bleuâtre glacée et même glauque presque figée déjà [...] sa voix aussi comme décolorée blanche neutre calme trop calme comme de la pierre. (HIS, 384-385; nous soulignons)

L'auteur de Histoire semble prendre au pied de la lettre la notion de "petite mort » dans l'acte sexuel. La métaphore devient chair. La mort in potentia contenue dans l'inertie, semble contrarier le concept de Vareille, l' « énergétique de l'immobile » ${ }^{25}$. Chez Simon, toute cette « énergie condensée, densifiée, hypertrophiée, amenée à l'état explosif » dont parle Vareille, ne culmine pourtant pas dans une explosion spectaculaire, mais plutôt dans une implosion silencieuse, vidant le corps de l'intérieur et ne laissant à l'extérieur qu'une carapace de carne, sclérosée dans l'état du (dernier) repos. Les amants se pétrifient de leur vivant. L'amant qui s'endort dans « le noir, l'immobilité corps étendu déjà fait de terre d'argile obscure » (HIS, 413), respirant encore, mais « exhalant la senteur immobile de la terre » $(H I S, 414)$, ne diffère point des dépouilles des cavaliers sous forme de « tas brun jaunâtre par terre qui ne bougeaient pas » $(F L A, 152)$. Les mouvements respiratoires des soldats s'amenuisent jusqu'à la limite du perceptible, «les poumons pleins de terre immobile $[\ldots]$ se vidant et se remplissant de terre » $(H I S, 415)$ pour la dernière fois. Cessation de toute activité, silence, agonie ; tel est l'appel du néant chtonien chez Simon.

\section{CONCLUSION}

En guise de clôture du présent article, reprenons les paroles de Jean-Yves Laurichesse qui constate : " $[\mathrm{N}] \mathrm{ul}$ ne peut ignorer la dimension terrienne de l'œuvre de Claude Simon $»^{26}$, et surtout dans le contexte de la mort. L'auteur

24 Ibidem (nous soulignons).

25 J.-C. Vareille, op. cit., p. 123.

26 J.-Y. Laurichesse, op. cit., p. 38. 
du Palace dépeint la fusion de l'organisme vivant avec la terre dans les termes d'un retour - parfois naturel, mais plus souvent accéléré - à la matrice originelle. Rare est la situation où la réintégration substantielle à l'élément s'opère de manière paisible ; la mort ainsi conjurée paraîtrait presque comme un choix et non pas une résignation à l'inévitable. D'habitude, elle est une force violente qui s'empare, de façon directe ou indirecte, des corps encore vivants et pourtant déjà perçus comme des dépouilles. En effet, l'image du tellurique anthropophage prévaut sur celle de la Terre-Mère nourricière, motif si cher à Bachelard.

Dans l'amour comme dans la guerre et les travaux agricoles, les personnages simoniens se voient stigmatisés par le chtonien, soit par la couleur terreuse de leur peau, soit par l'immobilité minérale de leur corps. Les héros des romans du corpus, soldats ou ouvriers, hommes ou femmes, entendent tous l'appel funèbre de l'élément, mais s'y soumettent de façon différente. La mort, fréquemment visualisée dans le phénomène de la pétrification, s'étend sur le monde entier afin de le piéger dans une inertie pré-mortuaire. Tout mouvement devient éphémère et illusoire, comme s'il ne s'agissait, au fond, que d'une forme d'《 inertie active », à l'instar de ces « quatre cavaliers et les cinq chevaux somnambuliques [...] non pas avançant mais levant et reposant les pieds sur place pratiquement immobiles sur la route, la carte la vaste surface de la terre les prés les bois se déplaçant lentement sous et autour d'eux » (FLA, 285). L'apocalypse à la Simon se déroule au ralenti, sans trompettes ni flammes, encore plus terrifiante peut-être car à peine visible et audible. La mort assaille sa victime de tous côtés, s'insinue imperceptiblement sous sa peau et l'ensevelit vivante sous la couche épaisse de l'herbe.

\section{THE CALL OF NOTHINGNESS : CLAUDE SIMON AND A TELLURIC TYPE OF DEATH}

Summary

The article proposes the analysis of different types of agony suffered by living beings as a result of contact with the element of Earth. The study is based on a corpus composed of seven novels by Claude Simon written after 1957, in a period of his fascination with formal experiments, and published by the Éditions de Minuit. The paper focuses on the representation of human body exposed to an evil influence of the Earth as element. Earth is considered as an anti-maternal force and a major destructive agent in Simon's universe. The reflections on the telluric character of death are inspired, among others, by Gaston Bachelard's thematic criticism and his conception of the four elements.

Key words: Claude Simon, new novel, death, four elements, Earth, Gaston Bachelard. 